OPEN ACCESS

Edited by:

Luis Sobrevia,

Pontificia Universidad Católica

de Chile, Chile

Reviewed by:

Claudio de Lucia,

Temple University, United States

Gerardo García-Rivas,

Monterrey Institute of Technology

and Higher Education (ITESM),

Mexico

*Correspondence: Gianluca Campo cmpglc@unife.it

Specialty section

This article was submitted to Clinical and Translational Physiology, a section of the journal

Frontiers in Physiology

Received: 04 October 2018 Accepted: 20 February 2019

Published: 11 March 2019

Citation:

Cimaglia P, Vieceli Dalla Sega F,

Vitali F, Lodolini V, Bernucci D,

Passarini G, Fortini F, Marracino L,

Aquila G, Rizzo P, Ferrari R and

Campo G (2019) Effectiveness of a

Novel Nutraceutical Compound

Containing Red Yeast Rice,

Polymethoxyflavones

and Antioxidants in the Modulation of Cholesterol Levels in Subjects With

Hypercholesterolemia and Low-Moderate Cardiovascular

Risk: The NIRVANA Study.

Front. Physiol. 10:217.

doi: 10.3389/fphys.2019.00217

\section{Effectiveness of a Novel} Nutraceutical Compound Containing Red Yeast Rice, Polymethoxyflavones and Antioxidants in the Modulation of Cholesterol Levels in Subjects With Hypercholesterolemia and Low-Moderate Cardiovascular Risk: The NIRVANA Study

Paolo Cimaglia', Francesco Vieceli Dalla Sega ${ }^{2}$, Francesco Vitali', Veronica Lodolini', Davide Bernucci', Giulia Passarini', Francesca Fortini' ${ }^{2}$, Luisa Marracino ${ }^{3}$, Giorgio Aquila ${ }^{3}$, Paola Rizzo ${ }^{2}$, Roberto Ferrari ${ }^{1,2}$ and Gianluca Campo ${ }^{1,2 *}$

\section{${ }^{1}$ Cardiovascular Institute, Azienda Ospedaliero-Universitaria di Ferrara, Ferrara, Italy, ${ }^{2}$ Maria Cecilia Hospital, GVM Care \& Research, Cotignola, Italy, ${ }^{3}$ Department of Medical Sciences, University of Ferrara, Ferrara, Italy}

Background: Red yeast rice supplements are broadly accepted as treatment for dyslipidaemia in subjects without high cardiovascular (CV) risk. Their effect on lipid profile is well known, but few data are available on their effect on endothelial function.

Objectives: To study the effect of a novel nutraceutical compound (NC) containing low monacolin $\mathrm{K}$ dose, polymethoxyflavones and antioxidants on lipid profile, endothelial function and oxidative stress.

Methods: Fifty-two subjects with low-moderate CV risk and dyslipidaemia (according to European guidelines) were enrolled and treated for 8 weeks with the NC. Blood samples were collected at baseline and at the end of treatment to assess changes in lipid profile, endothelial function and oxidative stress. The primary endpoint was the reduction of low density lipoprotein (LDL) cholesterol. Endothelial function was assessed through measurement of rate of apoptosis and nitric oxide (NO) production in human umbilical vein endothelial cells (HUVECs) treated with subject's serum. High-sensitivity C-reactive protein, 4-hydroxynonenal (HNE) and oxidized LDL (oxLDL) were markers of oxidative stress.

Results: Fifty subjects completed the study. The treatment caused a significant decrease in LDL $(-15.6 \%, p<0.001)$, oxLDL $(-21.5 \%, p<0.001)$, total cholesterol (TC), triglycerides, and ApoB. Apoptosis rate of HUVECs significantly decreased 
$(-15.9 \%, p<0.001)$. No changes were noted for NO levels and 4-HNE protein adducts. The reduction of the apoptosis rate was correlated to the reduction of oxLDL.

Conclusion: An 8-week treatment based on a novel NC containing low manocolin $\mathrm{K}$ dose, polymethoxyflavones and antioxidants improved lipid profile in subjects with dyslipidaemia and low-moderate CV risk. Secondarily, we observed an improvement in surrogate markers of endothelial function that may result from the reduction of oxLDL (Registered at www.clinicaltrials.gov, NCT03216811).

Keywords: red yeast rice, nutraceutical compound, hypercholesterolemia, cardiovascular prevention, endothelial function

\section{INTRODUCTION}

Dyslipidaemia represents an important CV risk factor. Reduction of low-density lipoprotein cholesterol (LDL) leads to a reduction of major adverse cardiovascular events (MACE), independently of the drug used (Silverman et al., 2016).

In primary prevention, according to international guidelines, a healthy lifestyle represents the first approach to dyslipidaemia, in case of unsuccess, drugs are needed. When drugs are needed, statins are the lipid-lowering treatment of choice due to the large evidence of efficacy and safety in reducing both LDL and MACE (Cholesterol Treatment Trialists' (CTT) Collaboration et al., 2010; Cholesterol Treatment Trialists' (CTT) Collaborators et al., 2012). However, statins are cardioprotective even in the presence of normal LDL cholesterol levels due to their pleiotropic effect (Sever et al., 2003; Oesterle et al., 2017). In the last few years, red yeast rice NC (containing monacolin $\mathrm{K}$ ) have been accepted as possible alternatives to statins (Pirro et al., 2016; Johnston et al., 2017; Poli et al., 2018; Zhang et al., 2018). Current European guidelines state that NC may be considered in individuals with elevated plasma cholesterol concentrations who do not qualify for drug treatment with statins in view of their global CV risk (Catapano et al., 2016). NC are also an alternative for subjects with intolerance to or not willing to take statins (Banach et al., 2018). Several NC are available on the market, and the common denominator is monacolin $\mathrm{K}$, whose activity on 3-hydroxy-3-methylglutaryl coenzyme A (HMG-CoA) reductase leads to the reduction of endogenous synthesis of cholesterol. Beside monacolin K, NC contain other molecules (e.g., berberin, policosanols, plant sterols, vitamins, and antioxidants) in various concentrations and compositions. These cofactors have lipidlowering activities that are in synergy with monacolin $\mathrm{K}$ action, and the effectiveness of the compounds is generally assessed in term of LDL reduction. Few studies investigated the effect of some compounds on endothelial function, through in vivo testing (Affuso et al., 2010; Trimarco et al., 2015; Cicero et al., 2016a,b, 2017; Esposito et al., 2018), analysis of endothelial damage biomarkers (Cicero et al., 2013; Derosa et al., 2014; Hermans et al., 2017) or in vitro experiments (Lin et al., 2011).The NC to optimize cholesterol, endothelial and inflammatory parameters

Abbreviations: CV, cardiovascular; HNE, Hydroxynonena; HUVECs, human umbilical vein endothelial cells; LDL, low density lipoprotein; NC, nutraceutical compound; NO, nitric oxide; oxLDL, oxidized LDL; SCORE, systemic coronary risk estimation. in subjects with hypercholesterolemia and low to moderate $\mathrm{CV}$ risk (NIRVANA) study was designed to test the effect of a novel NC in individuals with dyslipidaemia and low to moderate $\mathrm{CV}$ risk, in terms of reduction of cholesterol levels, but also in terms of improvement of endothelial function, and oxidative stress. The main novelty of the tested composition, compared to others, is the presence of polymethoxyflavones from tangerine extract, phenolic acids and flavonoids from Ipomoea batatas (sweet potato) extract, and hydroxytyrosol from olive fruit extract.

Polymethoxyflavones (citrus flavonoids) have antiinflammatory and anti-atherosclerosis activities (Li et al., 2009), while phenolic compounds from Ipomoea batatas, and hydroxytyrosol have antioxidant activity (Wang et al., 2016; Tejada et al., 2017). It is conceivable that the single components of the combination, by acting with different mechanism of action, could have a beneficial role on endothelial function.

Another important aspect of the tested NC is the dosage of monacolin $\mathrm{K}$ (3 mg), which was significantly lower than the $10 \mathrm{mg}$ dose recommended in 2011 by the (European Food Safety Authority (EFSA), 2011). Such a low dose of monacolin K has been proven effective in the reduction of LDL (Heinz et al., 2016), and it is potentially safer than the $10 \mathrm{mg}$ dose. This is a relevant aspect given the recent scientific opinion by EFSA on the safety of monacolins in red yeast rice (Younes et al., 2018).

\section{MATERIALS AND METHODS}

The NIRVANA is an investigator-initiated, prospective, singlecenter, interventional study. The Ethical Committee of Ferrara approved the study protocol in June 2017. All subjects gave written informed consent to participate. The study is registered at clinicaltrials.gov with the identifier NCT03216811.

\section{Inclusion and Exclusion Criteria}

Inclusion criteria were: (i) age > 18 years; (ii) ability to provide informed written consent; (iii) low-moderate CV risk according to SCORE (Piepoli et al., 2016), plus one of the following criteria (based on European guidelines on dyslipidaemia): $\mathrm{LDL}>190 \mathrm{mg} / \mathrm{dl}$ if SCORE $<1 \%$ or LDL $>100 \mathrm{mg} / \mathrm{dl}$ if SCORE 1-4\% (Catapano et al., 2016). Exclusion criteria were: high CV risk (SCORE $\geq 5 \%$ ), known CV disease, diabetes, creatinine clearance $<60 \mathrm{ml} / \mathrm{min}$, familial hypercholesterolemia, treatment 
with any lipid-lowering product in the previous 4 weeks, any concomitant chronic disease, intolerance to NC, high alcohol consumption, pregnancy, and breastfeeding.

\section{Study Design}

Subjects were recruited between June and October 2017 in the Prevention Center of the University of Ferrara (Ferrari, 2017; Ferrari and Guardigli, 2017, 2018; Ferrari and Cimaglia, 2018a,b; Ferrari et al., 2018). During the screening visit, study physicians assessed the patients' eligibility and widely discussed CV primary prevention and the importance of lifestyle changes concerning smoking, physical activity and a healthy diet. After the screening visit, a 4-week run-in period was started. During this period, subjects were strongly encouraged to follow the suggestions and the recommendations discussed in the screening visit. At the inclusion visit, eligibility was reassessed. If inclusion and exclusion criteria were satisfied, the subject was included and the NC was administered. After 8 weeks, the end of study visit was performed. During screening, inclusion and end of treatment visits the following parameters were assessed: blood pressure, heart rate, body weight, waist and hip circumferences.

\section{Nutraceutical Compound}

The NC tested was Cardiovis ${ }^{\circledR}$ Colesterolo (Bios Line, Padova, Italy). Each capsule consisted of red yeast rice containing $3 \mathrm{mg}$ of monacolin $\mathrm{K}$, polymethoxyflavones from tangerine extract (mainly nobiletin and tangeretin), hydroxytyrosol from olive fruit extract, phenolic acid, and flavonoids from Ipomoea batatas extract, vitamin E and coenzyme (Table 1).

Before production, titration of monacolin $\mathrm{K}$ in the red rice was checked by an external company. Furthermore, the quality of each lot was determined in terms of bacterial, yeasts and molds contamination.

All subjects included in the study received a single daily dose, that was prescribed to be taken in the evening. Treatment adherence was assessed by counting the number of pills returned at the end of study visit.

\section{Blood Samples}

Venous blood samples were collected at baseline (before starting treatment) and at the 8-week visit from an antecubital vein using a 21-gauge needle. All subjects underwent blood sampling after a 12-h fasting. TC, high-density lipoprotein (HDL), triglycerides, apolipoprotein A1 (ApoA1), apolipoprotein B100 (ApoB), creatinine, liver aminotransferases, creatinine phosphokinase and high-sensitivity c-reactive protein (hs-CRP)

TABLE 1 | Chemical composition of a capsule of nutraceutical compound.

Red rice fermented with Monascus purpureus titrated to $3 \mathrm{mg}$

$3 \%$ in monacolin $\mathrm{K}$

Tangerine extract titrated to $60 \%$ in polymethoxyflavones

Olive fruit extract titrated to $12 \%$ in hydroxytyrosol

Ipomoea batatas extract

Vitamin $\mathrm{E}$

Coenzyme Q10 were measured by standard enzymatic techniques. The LDL levels were calculated according to the Friedewald formula (Friedewald et al., 1972).

\section{Endothelial Function}

The endothelial function was evaluated measuring two surrogate markers of endothelial function: rate of apoptosis and NO production in HUVECs treated with serum from subjects as previously described (Campo et al., 2017; Pavasini et al., 2017; Vieceli Dalla Sega et al., 2018). Briefly, HUVECs were incubated for $48 \mathrm{~h}$ with growth media containing $20 \%$ serum from subjects. The rate of apoptosis was determined by flow cytometry after staining the cells with annexin $\mathrm{V}$ and propidium iodide. It was expressed as percentage (\%) of annexin $\mathrm{V}$ positive cells compared to the total number of cells. Each experiment was performed in triplicate and rate of apoptosis was the mean of the three experiments. The levels of NO were measured with the fluorogenic NO probe DAF-FM-DA. Fluorescence was quantified by flow cytometry and the NO levels were expressed as mean fluorescence.

\section{Oxidative Stress}

The oxidative stress was assessed by measuring oxLDL and 4HNE protein adduct in serum. OxLDL was measured by ELISA (oxLDL ELISA oxidized human, Cell Biolabs) according to the manufacturer's indication. The amount of oxLDL was expressed as arbitrary unit per $\mathrm{ml}$ serum. 4-HNE protein adduct was quantified by ELISA (Oxiselect ${ }^{\mathrm{TM}} \mathrm{HNE}$, Cell Biolab-competitive ELISA kit) according to the manufacturer's instructions. $50 \mu \mathrm{L}$ of serum was added to each well of the HNE conjugate coated plate, and the amount of 4-HNE protein adduct was expressed as $\mu \mathrm{g} / \mathrm{ml}$ equivalent to the 4-HNE bovine serum albumin (BSA) standard.

\section{Outcome}

The primary outcome was a change in the LDL cholesterol values after 8 weeks of treatment with the NC. Secondary outcomes included 8-week change of: TC, HDL, triglycerides, non-HDL cholesterol, Apo A1, ApoB, hs-CRP, oxLDL, 4-HNE, rate of HUVECs apoptosis, and NO levels. All subjects who were enrolled in the study and receiving at least one dose of the compound were included in the safety analyses. Any potential side effect was recorded.

\section{Statistical Analysis}

Based on previous studies, we assumed that the treatment would decrease LDL by $10 \%$. Accordingly, at least 40 subjects were required for $80 \%$ power and a two-sided-value of 0.05 . Continuous data were tested for normal distribution with the Kolmogorov-Smirnov test. The variables normally distributed were presented as mean $\pm \mathrm{SD}$, otherwise as median and interquartile range (IQR). For the comparison of parameters before and after treatment, the differences were tested for normality. Paired $t$-test or Wilcoxon test were used as appropriate. Of note, the LDL values (primary outcome) were normally distributed. Categorical variables were summarized in terms of numbers and percentages 
and were compared by using the two-sided Fisher's exact test. To better describe the response to NC, we prespecified subgroups (sex: male vs. female; metabolic syndrome as defined by Alberti et al. (2009): yes vs. no; c-reactive protein: $<$ vs. $\geq 2 \mathrm{mg} / \mathrm{L}$; baseline $\mathrm{LDL}:<$ vs. $\geq$ the median value; waist-hip ratio $(\mathrm{WHR}):<$ vs. $\geq$ World Health Organization cut-offs) where the LDL change was analyzed. A $p$-value was considered significant if $<0.05$. All analyses

TABLE 2 | Characteristics of the study population.

\begin{tabular}{|c|c|c|}
\hline & $\begin{array}{c}\text { Screened } \\
\text { population } \\
(n=117)\end{array}$ & $\begin{array}{c}\text { Study } \\
\text { population } \\
(n=52)\end{array}$ \\
\hline \multicolumn{3}{|l|}{ Clinical characteristics } \\
\hline Age (years) & $54.1 \pm 9.6$ & $55.7 \pm 4.8$ \\
\hline Male sex, no. (\%) & $73(62.4)$ & $23(44.2)$ \\
\hline BMI (Kg/m2) & $26.3 \pm 3.8$ & $25.8 \pm 4.0$ \\
\hline Hypertension, no. (\%) & $21(17.9)$ & $7(13.5)$ \\
\hline Family history of CVD, no. (\%) & $13(11.1)$ & $7(13.5)$ \\
\hline Current smoker, no. (\%) & $16(13.7)$ & $9(17.3)$ \\
\hline Previous smoker, no. (\%) & $41(35.0)$ & $16(30.8)$ \\
\hline Physical inactivity, no. (\%) & $31(26.5)$ & $14(26.9)$ \\
\hline Systolic BP (mmHg) & $122(115-130)$ & $125(120-130$ \\
\hline Diastolic BP (mmHg) & 75 (68-80) & $80(70-80)$ \\
\hline Heart rate (bpm) & $66.1 \pm 10.2$ & $64.5 \pm 9.7$ \\
\hline Waist circumference (cm) & $93.8 \pm 12.2$ & $92.2 \pm 11.9$ \\
\hline WHR & $\begin{array}{c}0.90 \\
(0.87-0.96)\end{array}$ & $\begin{array}{c}0.86 \\
(0.83-0.92)\end{array}$ \\
\hline Metabolic syndrome, no. (\%) & $37(31.6)$ & $16(30.8)$ \\
\hline \multicolumn{3}{|l|}{ Laboratory data } \\
\hline Total cholesterol (mg/dl) & $207.2 \pm 32.2$ & $246.2 \pm 27.1$ \\
\hline $\mathrm{HDL}(\mathrm{mg} / \mathrm{dl})$ & $57.0 \pm 13.3$ & $59.3 \pm 13.6$ \\
\hline Triglycerides (mg/dl) & $\begin{array}{c}95.0 \\
(70.0-131.0)\end{array}$ & $\begin{array}{c}101.0 \\
(75.5-163.5)\end{array}$ \\
\hline $\operatorname{LDL}(\mathrm{mg} / \mathrm{dl})$ & $128.9 \pm 27.5$ & $164.0 \pm 26.1$ \\
\hline Creatinine clearance (ml/min) & $\begin{array}{c}90.0 \\
(79.7-102.7)\end{array}$ & $\begin{array}{c}90.9 \\
(75.6-100.4)\end{array}$ \\
\hline Creatinine (mg/dl) & $0.89 \pm 0.13$ & $0.84 \pm 0.19$ \\
\hline \multicolumn{3}{|l|}{ 10-year cardiovascular risk } \\
\hline \multicolumn{3}{|l|}{ SCORE } \\
\hline$<1 \%$, no. $(\%)$ & $26(22.2)$ & $9(17.3)$ \\
\hline $1-5 \%$, no. $(\%)$ & $80(68.4)$ & $43(82.7)$ \\
\hline \multicolumn{3}{|l|}{ Relative risk } \\
\hline 1 no. (\%) & $68(58.1)$ & $14(26.9)$ \\
\hline 2 no. (\%) & 38 (32.5) & $23(44.2)$ \\
\hline$>2$ no. $(\%)$ & $10(8.5)$ & $15(28.9)$ \\
\hline \multicolumn{3}{|l|}{ Cardiovascular therapy } \\
\hline Aspirin, no. (\%) & $3(2.6)$ & $4(7.7)$ \\
\hline ACEi/ARB, no. (\%) & $19(16.2)$ & $6(11.5)$ \\
\hline Beta-blocker, no. (\%) & $1(0.9)$ & $1(1.9)$ \\
\hline Other antihypertensive drugs, no. (\%) & $9(7.7)$ & $3(5.8)$ \\
\hline
\end{tabular}

The variables normally distributed are presented as mean $\pm S D$, otherwise as median (IQR). BMI, body mass index; CVD, cardiovascular disease; BP, blood pressure; WHR, waist-hip ratio; HDL, high density lipoprotein; $L D L$, low density lipoprotein; SCORE, systemic coronary risk estimation; ACEi, angiotensin converting enzyme inhibitor; $A R B$, angiotensin II receptor blocker. were performed with STATISTICA 8 (Statsoft Inc, Tulsa, Okla, United States).

\section{RESULTS}

From June to October 2017, 117 subjects were screened (Table 2). Fifty-nine (50\%) resulted not eligible or declined to participate. Fifty-eight subjects entered the 4 -week run-in period. At the end of the run-in period, 52 subjects satisfied all inclusion and exclusion criteria and were enrolled. NC was regularly taken by 50 subjects (Figure 1 and Table 2). Table 2 shows the main characteristics of study population. Mean age was $56 \pm 5$ years and $44 \%$ were men. Mean LDL was $164 \pm 26$ and $83 \%$ of subjects were at moderate risk according to the SCORE (1-4\% risk at 10 years). There were no significant differences of all clinical and laboratory parameters before and after the run-in period. As shown in Figure 1, only two (4\%) individuals did not complete the 8 -week treatment period. One person stopped treatment for myalgia and one withdrew the consent for personal reasons. Weight, waist circumference and blood pressure did not change during the treatment period (data not shown).

\section{Lipid Profile}

Table 3 shows the effect of treatment on the lipid profile. After 8 weeks there was a significant decrease of LDL $(-15.6 \%$, $p<0.001$ ) (Figure 2A), concomitant with a significant decrease

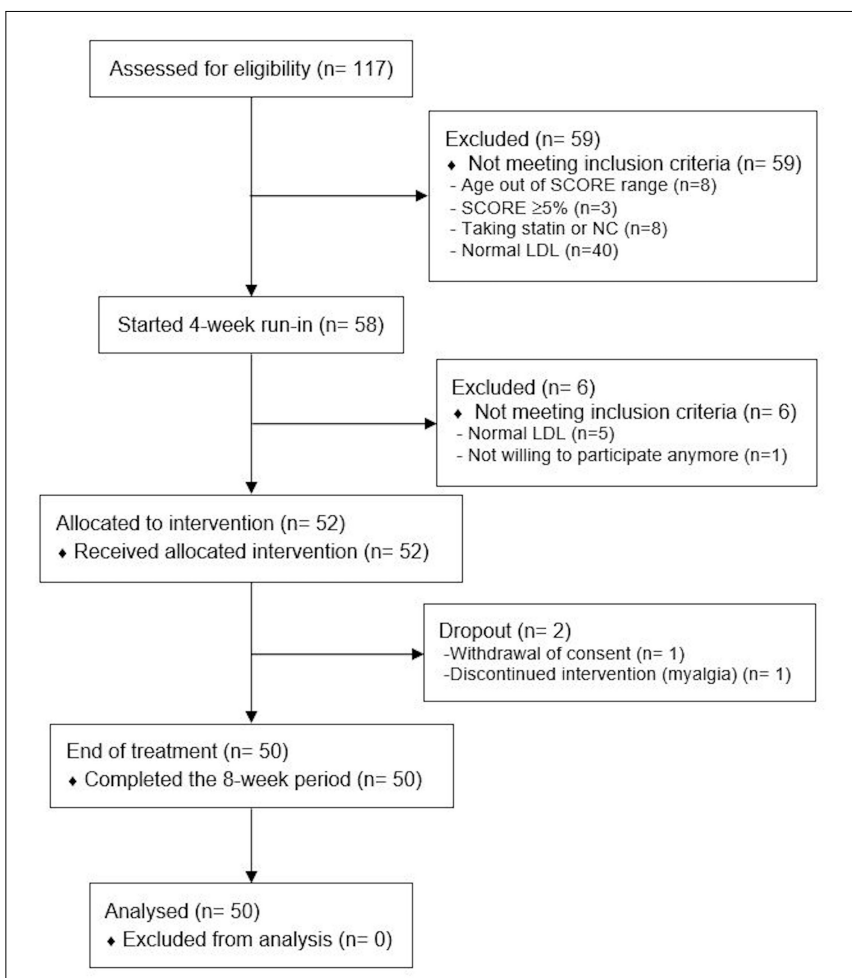

FIGURE 1 | Study flow-chart. SCORE, systemic coronary risk estimation; NC, nutraceutical compound; LDL, low density lipoprotein. 

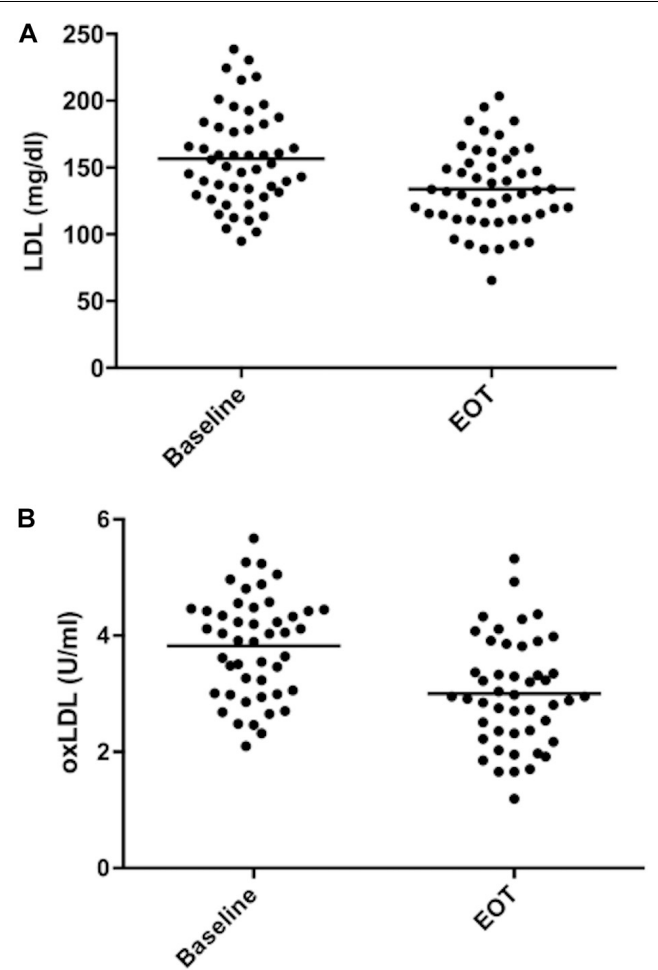

FIGURE 2 | Low density lipoprotein and oxidized low density lipoprotein values during the study. (A) LDL values. (B) oxidized LDL values. Mean values are shown as bars. EOT, end of treatment.

of TC, non-HDL cholesterol, triglycerides, ApoB, and five different lipoprotein ratios (Table 3). There was also a significant increase in ApoA1 $(+2.1 \%, p=0.01)$ and a trend for HDL raising $(+3.2 \%, p=0.057)($ Table 3$)$.

\section{Prespecified Subgroups Analysis}

Figure 3 shows the differences in LDL reduction among the subgroups. The median value of baseline LDL was $152.8 \mathrm{mg} / \mathrm{dl}$. The response to NC was stronger in the subgroup with higher baseline LDL compared to subjects with lower values $(-19.7 \%$ vs. $-9.1 \%, p=0.009)$. Furthermore, subjects with normal values of WHR had a greater LDL reduction compared to subjects with abnormal values $(-18.2 \%$ vs. $-10.0 \% ; p=0.046)$. Even though there was a trend for a better response to $\mathrm{NC}$ in female and in subjects without metabolic syndrome, the differences in the other couples of subgroups were not statistically significant. The effect of the NC on triglycerides, HDL, ApoB and ApoA1 in different subgroups is shown in Supplementary Material.

\section{Endothelial Function}

The eight-week treatment with NC significantly reduced the rate of apoptosis in HUVECs $(-15.9 \%, p<0.001)$ (Table 4) but did not affect the NO levels $(-1.2 \%, p=0.6)$.

\section{Oxidative Stress}

The difference in 4-HNE protein adducts in the serum was not statistically different after the 8 -week treatment. OxLDL levels were lower both as absolute values $(-21.5 \%, p<0.001)$ (Figure 2B) and as oxLDL/LDL ratio $(-12.4 \%, p<0.001)$ (Table 4). There was a good correlation between the decrease in LDL and oxLDL $(r=0.438 ; p=0.003)$. The change in the rate of apoptosis from baseline to end of treatment was related to the change in both oxLDL $(r=0.302, p=0.041)$ and oxLDL/LDL ratio $(r=0.348, p=0.018)$. On the other hand, it did not correlate with the change in LDL values ( $r=0.003, p=0.983)$.

The analysis of variance (ANOVA) of the apoptosis rate change among quartiles of the oxLDL change was statistically significant ( $p$ for trend $=0.036$ ) (Figure 4).

TABLE 3 | Lipid profile and safety parameters before and after the treatment.

\begin{tabular}{|c|c|c|c|c|c|}
\hline LDL (mg/dl) & $\begin{array}{c}\text { Baseline }(\boldsymbol{n}=\mathbf{5 2}) \\
156.6 \pm 35.4\end{array}$ & $\begin{array}{l}\text { EOT }(\boldsymbol{n}=\mathbf{5 0}) \\
133.9 \pm 30.3\end{array}$ & \multicolumn{2}{|c|}{ Treatment difference } & $\frac{\boldsymbol{P}}{<0.001}$ \\
\hline TC (mg/dl) & $241.0 \pm 40.4$ & $216.4 \pm 36.2$ & $-24.7 \pm 23.9$ & $-10.2 \%$ & $<0.001$ \\
\hline Triglycerides (mg/dl) & $107.5(82.3-171.3)$ & $100.5(78.0-148.3)$ & $-13.5(-40.5-18.25)$ & $-12.6 \%$ & 0.032 \\
\hline nonHDL cholesterol (mg/dl) & $185.0 \pm 39.0$ & $159.1 \pm 35.2$ & $-26.5 \pm 24.3$ & $-14.3 \%$ & $<0.001$ \\
\hline $\mathrm{TC} / \mathrm{HDL}$ & $4.6 \pm 1.3$ & $4.0 \pm 1.0$ & $-0.68 \pm 0.72$ & $-14.8 \%$ & $<0.001$ \\
\hline LDL/HDL & $2.9 \pm 0.9$ & $2.5 \pm 0.7$ & $-0.5 \pm 0.5$ & $-17.2 \%$ & $<0.001$ \\
\hline AIP & $0.01 \pm 0.36$ & $-0.06 \pm 0.28$ & $-0.07 \pm 0.21$ & $-7 \%$ & 0.025 \\
\hline ApoB (mg/dl) & $112.2 \pm 21.1$ & $94.3 \pm 19.5$ & $-18.5 \pm 13.1$ & $-16.5 \%$ & $<0.001$ \\
\hline ApoA1 (mg/dl) & $142.7 \pm 17.9$ & $144.6 \pm 17.4$ & $3.0 \pm 7.9$ & $2.1 \%$ & 0.01 \\
\hline ApoB/ApoA1 & $0.80 \pm 0.19$ & $0.66 \pm 0.17$ & $-0.15 \pm 0.09$ & $-18.8 \%$ & $<0.001$ \\
\hline AST (U/L) & $22.4 \pm 4.9$ & $23.3 \pm 6.2$ & $0.9 \pm 3.9$ & $4 \%$ & 0.103 \\
\hline
\end{tabular}

The variables normally distributed are presented as mean $\pm S D$, otherwise as median (IQR). EOT, end of treatment; AIP, atherogenic index of plasma [log(triglycerides/HDL)]. 


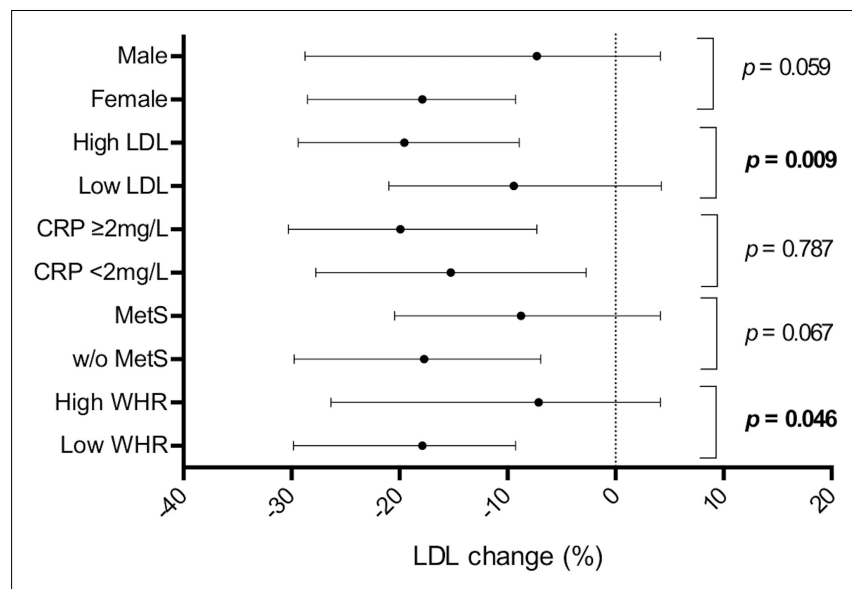

FIGURE 3 | Low density lipoprotein reduction in different subgroups. LDL reduction is shown as median and interquartile range. $P$-values refer to the difference in the subgroups shown on the left. CRP, c-reactive protein; MetS, metabolic syndrome; WHR, waist-hip ratio.

\section{Safety}

Nutraceutical compound was well tolerated and did not cause any change in creatinine, liver enzymes and creatine kinase values (Table 3 ).

\section{DISCUSSION}

Several NC based on red yeast rice with different dosages of monacolin $\mathrm{K}$ and other cofactors are available on the market. However, not of all them have been proven effective by proper scientific studies. This is relevant considering the variability in the quantity of monacolin $\mathrm{K}$ in 28 different red yeast rice supplements available in the United States of America (Cohen et al., 2017). When tested, the efficacy of these NC is determined

TABLE 4 | Parameters regarding endothelial function and oxidative stress.

\begin{tabular}{lccccc}
\hline & $\begin{array}{c}\text { Baseline } \\
(\boldsymbol{n}=\mathbf{5 2})\end{array}$ & $\begin{array}{c}\text { EOT } \\
(\boldsymbol{n}=\mathbf{5 0})\end{array}$ & Treatment difference & $\boldsymbol{P}$ \\
\hline $\begin{array}{l}\text { Apoptosis rate } \\
(\%)\end{array}$ & $6.9 \pm 1.6$ & $5.8 \pm 1.3$ & $-1.1 \pm 1.0$ & $-15.9 \%$ & $<0.001$ \\
$\begin{array}{l}\text { Nitric oxide } \\
\text { (FAU) }\end{array}$ & $25.7 \pm 4.6$ & $25.4 \pm 4.6$ & $-0.3 \pm 4.1$ & $-1.2 \%$ & 0.626 \\
$\begin{array}{l}\text { 4-HNE (U/ml) } \\
\text { oxLDL (U/ml) }\end{array}$ & $30.9 \pm 51.8$ & $78.6 \pm 54.2$ & $7.7 \pm 30.3$ & $10.9 \%$ & 0.089 \\
$\begin{array}{l}\text { oxLDL/LDL } \\
(\mathrm{U} / \mathrm{mg})\end{array}$ & $2.51 \pm 0.54$ & $2.20 \pm 0.61$ & $-0.31 \pm 0.48$ & $-12.4 \%$ & $<0.001$ \\
$\begin{array}{l}\text { oxLDL/ApoB } \\
(\mathrm{U} / \mathrm{mg})\end{array}$ & $3.46 \pm 0.72$ & $3.22 \pm 0.93$ & $-0.21 \pm 0.75$ & $-6.0 \%$ & 0.067 \\
$\begin{array}{l}\text { oxLDL/HDL } \\
(\mathrm{U} / \mathrm{mg})\end{array}$ & $7.53 \pm 2.72$ & $5.71 \pm 2.40$ & $-1.82 \pm 1.70$ & $-24.2 \%$ & $<0.001$ \\
hs-CRP (mg/L) & $1.2(0.5-2.0)$ & $1.4(0.7-2.2)$ & $0.05(-0.23-0.5)$ & $4.2 \%$ & 0.301 \\
\hline
\end{tabular}

The variables normally distributed are presented as mean $\pm S D$, otherwise as median (IQR). EOT, end of treatment; FAU, fluorescence arbitrary units; hs-CRP, high sensitivity C-reactive protein. by reduction of LDL cholesterol. Our study shows that the tested $\mathrm{NC}$ containing low monacolin $\mathrm{K}$ dose, polymethoxyflavones and antioxidants significantly reduced the LDL cholesterol after 8 weeks of treatment, thus confirming the previous studies (Ruscica et al., 2014; Mazza et al., 2015; Heinz et al., 2016; Pirro et al., 2016; Barrios et al., 2017). Our data also confirm the evidence that subjects with higher baseline values of LDL have the greatest benefit from NC treatment (Heinz et al., 2016).

The main strengths and novelties of our findings can be summarized as follows. First, we demonstrated that a NC with monacolin $\mathrm{K}$, beside the reduction of $\mathrm{LDL}$, improves endothelial function assessed in vitro as a reduction of the rate of apoptosis in HUVECs incubated with serum from treated subjects, a methodology that was previously validated and related to clinical outcomes (Agnoletti et al., 1999; Valgimigli et al., 2003; Campo et al., 2017; Pavasini et al., 2017). Enhanced endothelial apoptosis is supposed to be the initial step in the development of atherosclerosis. It is considered the underlying mechanism of the pleiotropic effects of both statins and ACE inhibitors, which have been shown to reduce endothelial apoptosis and increase endothelial NO production (Ceconi et al., 2007; Oesterle et al., 2017). In our study the NO levels in HUVECs were not significantly increased by the treatment with the tested NC. There are several explanations for this. The low-risk subjects enrolled in our study could have just a mild endothelial dysfunction with initial increase of the rate of apoptosis, but without significant impairment of endothelial NO production that could not be further improved by treatments, thus maintaining normal or near normal endothelial function. Furthermore, NO levels depend on cumulative effects of different factors, and endothelial cells apoptosis and NO are controlled by different pathways. Therefore it is not surprising that variations of serum cytokines or lipids may modulate apoptosis or NO pathway differently. Alternatively, the treatment duration of 8 weeks could be too short to result in an improvement in NO production.

Second, dyslipidaemia is known to be associated with oxidative stress and high oxLDL levels (Ercan et al., 2014; Harmon et al., 2016). Our study demonstrated that NC reduced oxLDL both in terms of absolute values and oxLDL/LDL ratio. In contrast, treatment did not reduce 4-HNE total protein adducts, a biomarker of oxidative damage to lipids. Low physiological levels of 4-HNE have a pro-survival role on endothelial cells, while high doses of 4 -HNE result to be toxic (Chapple et al., 2013). Due to the lack of standardized data for 4-HNE adducts levels in serum, it is difficult to establish a 4 -HNE physiological threshold. In line with the results of NO, this may be related to the low risk of the population studied with moderate dyslipidemia insufficient to cause abnormal accumulation of 4-HNE. This hypothesis is supported by the findings in which baseline 4HNE levels do not correlate with LDL or other parameters related to dyslipidaemia and/or inflammation, suggesting that the majority of enrolled subjects could have 4-HNE physiological levels. The oxidation of LDL has been recognized as an early event in the progression of atherosclerosis, and the oxLDL levels are linearly associated with CV diseases (Trpkovic et al., 2015). It is known that apoptosis can be induced in endothelial cells by high levels of oxLDL via a mechanism mediated by the 

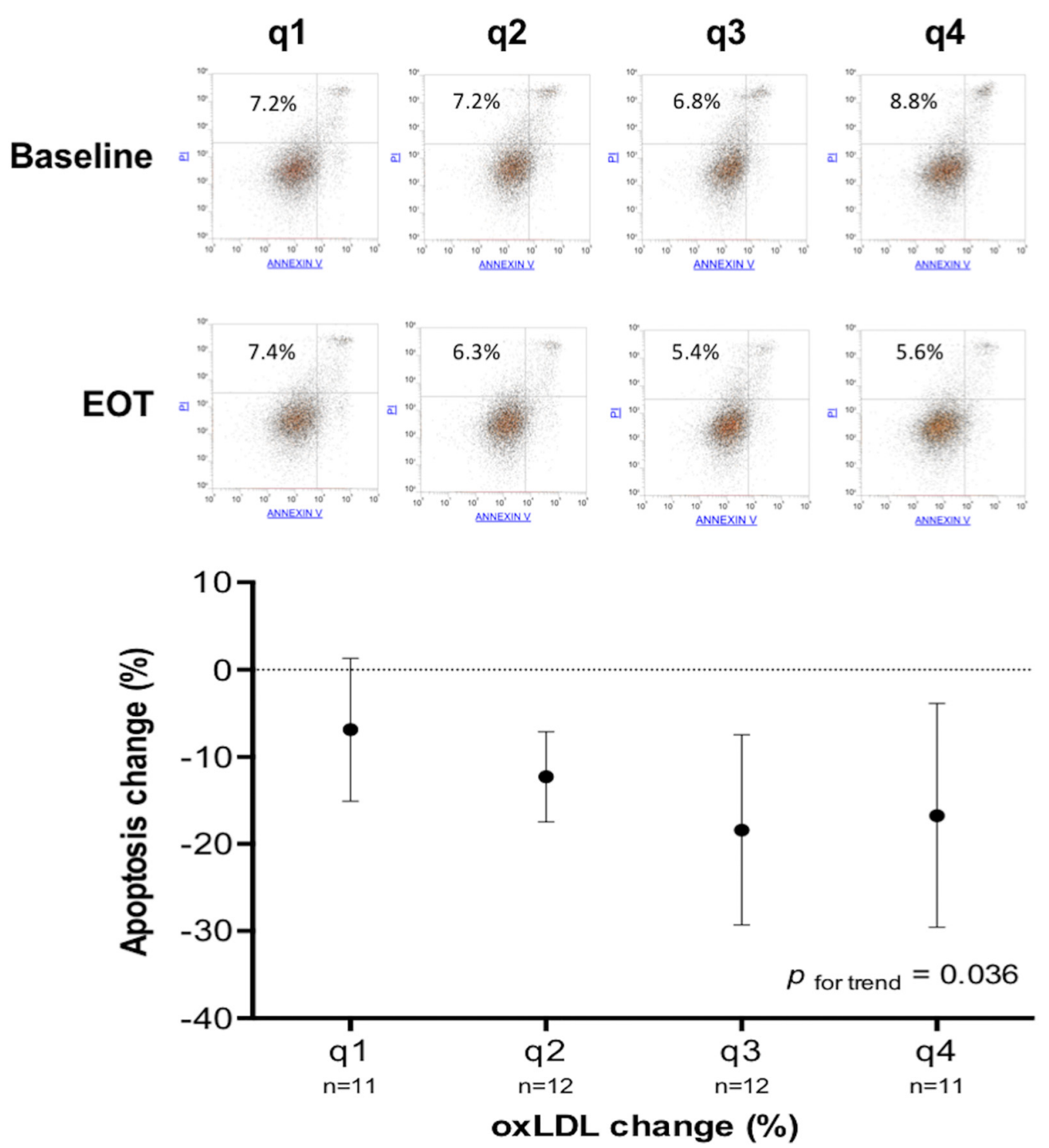

FIGURE 4 | Change of apoptosis rate according to quartiles of oxLDL change. In the main graph, for each quartile of oxLDL change ( $q 1>-8.78 \% ; q 4<-34.66 \%)$ is shown the mean and SD of apoptosis rate change. The upper part of the figure shows the flow cytometry and apoptosis rate (\%) before and after treatment of four representative subjects belonging to different quartiles of oxLDL change.

oxidized low-density lipoprotein receptor 1 (LOX1) (Valente et al., 2014) and carried out by classic caspases (Sata and Walsh, 1998). Indeed, we observed a correlation between the reduction of oxLDL and the reduction of apoptosis rate in HUVECs, suggesting that improvement in endothelial function may be mediated by a decrease in oxLDL. Hydroxytyrosol and vitamin $\mathrm{E}$ act as antioxidants protecting against LDL oxidation in humans (Najafpour Boushehri et al., 2012; Mateos et al., 2016), and nobiletin (polymethoxyflavone) and coenzyme Q10 can prevent endothelial cells apoptosis induced by oxLDL by modulating LOX-1 (Eguchi et al., 2006; Tsai et al., 2011). Overall, the effect on endothelial apoptosis is probably caused by the cumulative action on LDL lowering mediated by monacolin $\mathrm{K}$, and the protection from LDL oxidation provided by the nutraceutical antioxidants.
Third, to our knowledge this is the first study on the use of a NC that selected the subjects using the most recent European Society of Cardiology (ESC) guidelines on dyslipidaemia and primary CV prevention (Catapano et al., 2016; Piepoli et al., 2016). Indeed, the selection of individuals was made considering both LDL value and the absolute CV risk of the subject, the latter calculated through SCORE. In literature, there are only two studies that included a CV risk score (Framingham) as an inclusion criterium (Gonnelli et al., 2015; Derosa et al., 2017). In all the others, subjects were enrolled only according to LDL values, with minimum baseline LDL values for inclusion ranging between 115 and $150 \mathrm{mg} / \mathrm{dl}$ with, sometimes, an upper limit, from 160 to $230 \mathrm{mg} / \mathrm{dl}$. Choosing to start a preventive intervention based purely on a chemical or clinical value without considering the global risk of the subject could be non-optimal 
(Blood Pressure Lowering Treatment Trialists Collaboration, 2014; Stone et al., 2014; Catapano et al., 2016; Piepoli et al., 2016).

Fourth, unlike previous studies, we did not detect any change in hs-CRP. This can be explained with the lower dosage of monacolin $\mathrm{K}$ and coenzyme Q10 in our NC and, especially, with lower baseline hs-CRP values in our population. Indeed, when we performed a sub-analysis only in subjects with baseline hs-CRP greater than $2 \mathrm{mg} / \mathrm{L}[N=10,2.5$ $(2.1-3.5) \mathrm{mg} / \mathrm{L})]$, there was a significant reduction after the treatment $(-0.7 \pm 0.6 \mathrm{mg} / \mathrm{L}, p=0.008)$, in line with findings by Cicero et al. $(2013,2016 a)(\sim 20 \%)$.

Fifth, subgroups analysis shows that subjects with metabolic syndrome had a lower reduction of LDL compared to subject without the syndrome, even though the difference is not significant. Many NC have been tested with success in subjects with metabolic syndrome, but we are not aware of studies of comparison between these groups (Ruscica et al., 2014; Verhoeven et al., 2015; Mazza et al., 2018). This finding is confirmed by a significant lower response to NC in subjects with abnormal WHR, an index of abdominal adiposity associated with metabolic abnormalities and increased CV risk (Emdin et al., 2017). However, NIRVANA was not powered to test this hypothesis, hence these findings have an exploratory value. A possible explanation could be that low adherence to healthy lifestyle recommendations had caused both the metabolic abnormalities (i.e., high WHR and/or metabolic syndrome) and a lower response to NC.

\section{Study Limitations}

The present study has limitations. First, the absence of a control group; this decision was made because the efficacy of NC with $3 \mathrm{mg}$ of monacolin $\mathrm{K}$ vs. placebo has already been investigated. Second, the study has a relatively small sample size, even though it was powered for the primary endpoint. Third, an 8-week study period, although in line with other studies, is not adequate to evaluate the long-term efficacy of NC. Fourth, in the view of previous experience of our team, potential effect of the NC on endothelial function has been tested with an in vitro experiment, rather than in vivo tests or biomarkers analysis. We are aware that the two latter should be preferred to the first one. However, the experiment we used was previously validated, and related to clinical outcomes. Finally, subgroups analysis and results on endothelial function have to be considered hypothesis generation.

\section{REFERENCES}

Affuso, F., Ruvolo, A., Micillo, F., Saccà, L., and Fazio, S. (2010). Effects of a nutraceutical combination (berberine, red yeast rice and policosanols) on lipid levels and endothelial function randomized, double-blind, placebo-controlled study. Nutr. Metab. Cardiovasc. Dis. 20, 656-661. doi: 10.1016/j.numecd.2009. 05.017

Agnoletti, L., Curello, S., Bachetti, T., Malacarne, F., Gaia, G., Comini, L., et al. (1999). Serum from patients with severe heart failure downregulates eNOS and is proapoptotic: role of tumor necrosis factor-alpha. Circulation 100, 1983-1991. doi: 10.1161/01.CIR.100.19.1983
It follows that this is only a small "proof-of-concept," hypothesisgenerating study.

\section{Conclusion}

The NIRVANA study showed that 8-week treatment with a NC containing low monacolin $\mathrm{K}$ dose, polymethoxyflavones, hydroxytyrosol, vitamin E, coenzyme Q10 and Ipomoea batatas extract was able to reduce by $\sim 15 \%$ LDL levels in subjects with dyslipidaemia and low to moderate CV risk. In addition, it was associated with a significant improvement in surrogate markers of endothelial function, mainly mediated by a reduction of oxLDL.

\section{AUTHOR CONTRIBUTIONS}

PC, GC, FVDS, FV, and PR contributed to conception and design of the study. PC, FV, VL, DB, GP, FF, GA, and LM organized the database. PC, GC, and VL performed the statistical analysis. PC, FVDS, and GC wrote the first draft of the manuscript. RF and PR wrote sections of the manuscript. All authors contributed to manuscript revision, read, and approved the submitted version.

\section{FUNDING}

This study was partially supported by a research grant from Bios Line, which had no role in the collection, analysis, and interpretation of data; writing of the report; and the decision to submit the paper for publication.

\section{ACKNOWLEDGMENTS}

We thank the Bios Line company (Bios Line S.p.A., Ponte San Nicolò, Padova, Italy) for providing the pills containing Cardiovis ${ }^{\circledR}$ Colesterolo and for their technical support. We acknowledge the Fondazione Anna Maria Sechi per il Cuore (FASC), Italy, for the support in the management of the study.

\section{SUPPLEMENTARY MATERIAL}

The Supplementary Material for this article can be found online at: https://www.frontiersin.org/articles/10.3389/fphys. 2019.00217/full\#supplementary-material

Alberti, K. G., Eckel, R. H., Grundy, S. M., Zimmet, P. Z., Cleeman, J. I., Donato, K. A., et al. (2009). Harmonizing the metabolic syndrome: a joint interim statement of the international diabetes federation task force on epidemiology and prevention; national heart, lung, and blood institute; American heart association; world heart federation; international. Circulation 120, 1640-1645. doi: 10.1161/CIRCULATIONAHA.109.192644

Banach, M., Maria Patti, A., Vincenza Giglio, R., Cicero, A. F., Atanasov, A. G., Bajraktari, G., et al. (2018). The role of nutraceuticals in statin\&nbsp;intolerant patients. J. Am. Coll. Cardiol. 72, 96-118. doi: 10.1016/j.jacc.2018.04.040

Barrios, V., Escobar, C., Cicero, A. F. G., Burke, D., Fasching, P., Banach, M., et al. (2017). A nutraceutical approach (Armolipid Plus) to reduce total and LDL 
cholesterol in individuals with mild to moderate dyslipidemia: review of the clinical evidence. Atheroscler. Suppl. 24, 1-15. doi: 10.1016/j.atherosclerosissup. 2016.10.003

Blood Pressure Lowering Treatment Trialists Collaboration (2014). Blood pressure-lowering treatment based on cardiovascular risk: a meta-analysis of individual patient data. Lancet 384, 591-598. doi: 10.1016/S0140-6736(14) 61212-5

Campo, G., Dalla Sega, F., Pavasini, R., Aquila, G., Gallo, F., Fortini, F., et al. (2017). Biological effects of ticagrelor over clopidogrel in patients with stable coronary artery disease and chronic obstructive pulmonary disease. Thromb Haemost. 117, 1208-1216. doi: 10.1160/TH16-12-0973

Catapano, A. L., Graham, I., De Backer, G., Wiklund, O., Chapman, M. J., Drexel, H., et al. (2016). ESC/EAS guidelines for the management of dyslipidaemias. Eur. Heart J. 37, 29991-30581. doi: 10.1093/eurheartj/ehw272

Ceconi, C., Francolini, G., Bastianon, D., Gitti, G. L., Comini, L., and Ferrari, R. (2007). Differences in the effect of angiotensin-converting enzyme inhibitors on the rate of endothelial cell apoptosis: in vitro and in vivo studies. Cardiovasc. Drugs Ther. 21, 423-429. doi: 10.1007/s10557-007-6068-5

Chapple, S. J., Cheng, X., and Mann, G. E. (2013). Effects of 4-hydroxynonenal on vascular endothelial and smooth muscle cell redox signaling and function in health and disease. Redox Biol. 1, 319-331. doi: 10.1016/j.redox.2013.04.001

Cholesterol Treatment Trialists' (CTT) Collaboration, Baigent, C., Blackwell, L., Emberson, J., Holland, L. E., Reith, C., et al. (2010). Efficacy and safety of more intensive lowering of LDL cholesterol: a meta-analysis of data from 170000 participants in 26 randomised trials. Lancet 376, 1670-1681. doi: 10.1016/ S0140-6736(10)61350-5

Cholesterol Treatment Trialists' (CTT) Collaborators, Mihaylova, B., Emberson, J., Blackwell, L., Keech, A., Simes, J., et al. (2012). The effects of lowering LDL cholesterol with statin therapy in people at low risk of vascular disease: metaanalysis of individual data from 27 randomised trials. Lancet 380, 581-590. doi: 10.1016/S0140-6736(12)60367-5

Cicero, A., Morbini, M., Parini, A., Urso, R., Rosticci, M., Grandi, E., et al. (2016a). Effect of red yeast rice combined with antioxidants on lipid pattern, hs-CRP level, and endothelial function in moderately hypercholesterolemic subjects. Ther. Clin. Risk Manag. 12:281. doi: 10.2147/TCRM.S91817

Cicero, A. F., Morbini, M., Rosticci, M., D”Addato, S., Grandi, E., and Borghi, C. (2016b). Middle-term dietary supplementation with red yeast rice plus coenzyme Q10 improves lipid pattern, endothelial reactivity and arterial stiffness in moderately hypercholesterolemic subjects. Ann. Nutr. Metab. 68, 213-219. doi: 10.1159/000445359

Cicero, A. F. G., Derosa, G., Parini, A., Maffioli, P., D’Addato, S., Reggi, A., et al. (2013). Red yeast rice improves lipid pattern, high-sensitivity C-reactive protein, and vascular remodeling parameters in moderately hypercholesterolemic Italian subjects. Nutr. Res. 33, 622-628. doi: 10.1016/J. NUTRES.2013.05.015

Cicero, A. F. G., Fogacci, F., Bove, M., Veronesi, M., Rizzo, M., Giovannini, M., et al. (2017). Short-term effects of a combined nutraceutical on lipid level, fatty liver biomarkers, hemodynamic parameters, and estimated cardiovascular disease risk: a double-blind, placebo-controlled randomized clinical trial. $A d v$. Ther. 34, 1966-1975. doi: 10.1007/s12325-017-0580-1

Cohen, P. A., Avula, B., and Khan, I. A. (2017). Variability in strength of red yeast rice supplements purchased from mainstream retailers. Eur. J. Prev. Cardiol. 24, 1431-1434. doi: 10.1177/2047487317715714

Derosa, G., Bonaventura, A., Bianchi, L., Romano, D., D’Angelo, A., Fogari, E., et al. (2014). A randomized, placebo-controlled study on the effects of a nutraceutical combination of red yeast rice, silybum marianum and octasonol on lipid profile, endothelial and inflammatory parameters. J. Biol. Regul. Homeost. Agents 28, 317-324.

Derosa, G., D’Angelo, A., Romano, D., and Maffioli, P. (2017). Effects of a combination of berberis aristata, silybum marianum and monacolin on lipid profile in subjects at low cardiovascular risk; a double-blind, randomized, placebo-controlled trial. Int. J. Mol. Sci. 18:E343. doi: 10.3390/ijms18020343

Eguchi, A., Murakami, A., and Ohigashi, H. (2006). Nobiletin, a citrus flavonoid, suppresses phorbol ester-induced expression of multiple scavenger receptor genes in THP-1 human monocytic cells. FEBS Lett. 580, 3321-3328. doi: 10. 1016/j.febslet.2006.04.077

Emdin, C. A., Khera, A. V., Natarajan, P., Klarin, D., Zekavat, S. M., Hsiao, A. J., et al. (2017). Genetic association of waist-to-hip ratio with cardiometabolic traits, type 2 diabetes, and coronary heart disease. JAMA 317:626. doi: 10.1001/ jama.2016.21042

Ercan, M., Firtina, S., and Konukoglu, D. (2014). Comparison of plasma viscosity as a marker of endothelial dysfunction with nitric oxide and asymmetric dimethylarginine in subjects with dyslipidemia. Clin. Hemorheol. Microcirc. 57, 315-323. doi: 10.3233/CH-131706

Esposito, R., Sorrentino, R., Giugliano, G., Avvedimento, M., Paolillo, R., Santoro, C., et al. (2018). Different age-independent effects of nutraceutical combinations on endothelium-mediated coronary flow reserve. Immun. Ageing 15:30. doi: 10.1186/s12979-018-0138-3

European Food Safety Authority (EFSA) (2011). Scientific opinion on the substantiation of health claims related to monacolin $\mathrm{K}$ from red yeast rice and maintenance of normal blood LDL cholesterol concentrations (ID 1648, 1700) pursuant to Article 13(1) of Regulation (EC) No 1924/2006. EFSA J. 9:2304. doi: $10.2903 /$ j.efsa.2011.2304

Ferrari, R. (2017). Ferrara I: introduction. Eur. Heart J. 38, 3255-3257. doi: 10. 1093/eurheartj/ehx622

Ferrari, R., and Cimaglia, P. (2018a). Ferrara IV. Eur. Heart J. 39, 497-501. doi: 10.1093/eurheartj/ehy030

Ferrari, R., and Cimaglia, P. (2018b). Ferrara V. Eur. Heart J. 39, 977-981. doi: 10.1093/eurheartj/ehy084

Ferrari, R., and Guardigli, G. (2017). Ferrara II. Eur. Heart J. 38, 3337-3339. doi: 10.1093/eurheartj/ehx675

Ferrari, R., and Guardigli, G. (2018). Ferrara III. Eur. Heart J. 39, 88-90. doi: 10.1093/eurheartj/ehx756

Ferrari, R., Guardigli, G., and Cimaglia, P. (2018). Ferrara VI. Eur. Heart J. 39, 1509-1511. doi: 10.1093/eurheartj/ehy180

Friedewald, W. T., Levy, R. I., and Fredrickson, D. S. (1972). Estimation of the concentration of low-density lipoprotein cholesterol in plasma, without use of the preparative ultracentrifuge. Clin. Chem. 18, 499-502. doi: 10.1177/ 107424840501000106

Gonnelli, S., Caffarelli, C., Stolakis, K., Cuda, C., Giordano, N., and Nuti, R. (2015). Efficacy and tolerability of a nutraceutical combination (red yeast rice, policosanols, and berberine) in patients with low-moderate risk hypercholesterolemia: a double-blind, placebo-controlled study. Curr. Ther. Res. Clin. Exp. 77, 1-6. doi: 10.1016/j.curtheres.2014.07.003

Harmon, M. E., Campen, M. J., Miller, C., Shuey, C., Cajero, M., Lucas, S., et al. (2016). Associations of circulating oxidized LDL and conventional biomarkers of cardiovascular disease in a cross-sectional study of the navajo population. PLoS One 11:e0143102. doi: 10.1371/journal.pone.0143102

Heinz, T., Schuchardt, J. P., Möller, K., Hadji, P., and Hahn, A. (2016). Low daily dose of $3 \mathrm{mg}$ monacolin $\mathrm{K}$ from RYR reduces the concentration of LDL-C in a randomized, placebo-controlled intervention. Nutr. Res. 36, 1162-1170. doi: 10.1016/J.NUTRES.2016.07.005

Hermans, N., Van der Auwera, A., Breynaert, A., Verlaet, A., De Bruyne, T., Van Gaal, L., et al. (2017). red yeast rice-olive extract supplement reduces biomarkers of oxidative stress, OxLDL and Lp-PLA2, in subjects with metabolic syndrome: a randomised, double-blind, placebo-controlled trial. Trials 18, 1-8. doi: 10.1186/s13063-017-2058-5

Johnston, T. P., Korolenko, T. A., Pirro, M., and Sahebkar, A. (2017). Preventing cardiovascular heart disease: Promising nutraceutical and non-nutraceutical treatments for cholesterol management. Pharmacol. Res. 120, 219-225. doi: 10.1016/j.phrs.2017.04.008

Li, S., Pan, M. H., Lo, C. Y., Tan, D., Wang, Y., Shahidi, F., et al. (2009). Chemistry and health effects of polymethoxyflavones and hydroxylated polymethoxyflavones. J. Funct. Foods 1, 2-12. doi: 10.1016/j.jff.2008. 09.003

Lin, C., Lin, Y., Huang, P., Tsai, H., and Chen, Y. (2011). Inhibition of endothelial adhesion molecule expression by monascus purpureus-fermented rice metabolites, monacolin K, ankaflavin, and monascin. J. Sci. Food Agric. 91, 1751-1758. doi: 10.1002/jsfa.4371

Mateos, R., Martínez-López, S., Baeza Arévalo, G., Amigo-Benavent, M., Sarriá, B., and Bravo-Clemente, L. (2016). Hydroxytyrosol in functional hydroxytyrosolenriched biscuits is highly bioavailable and decreases oxidised low density lipoprotein levels in humans. Food Chem. 205, 248-256. doi: 10.1016/j. foodchem.2016.03.011

Mazza, A., Lenti, S., Schiavon, L., Di Giacomo, E., Tomasi, M., Manunta, R., et al. (2018). Effect of Monocolin K and COQ10 supplementation in 
hypertensive and hypercholesterolemic subjects with metabolic syndrome. Biomed. Pharmacother. 105, 992-996. doi: 10.1016/j.biopha.2018.06.076

Mazza, A., Lenti, S., Schiavon, L., Zuin, M., D’Avino, M., Ramazzina, E., et al. (2015). Nutraceuticals for serum lipid and blood pressure control in hypertensive and hypercholesterolemic subjects at low cardiovascular risk. $A d v$. Ther. 32, 680-690. doi: 10.1007/s12325-015-0229-x

Najafpour Boushehri, S., Yusof, R. M., Nasir Mohammad Taib, M., Mirzaei, K., Yazdekhasti, N., and Akbarzadeh, S. (2012). Effect of vitamin supplementation on serum oxidized low-density lipoprotein levels in male subjects with cardiovascular disease risk factors. Iran J. Basic Med. Sci. 15, 958-964.

Oesterle, A., Laufs, U., and Liao, J. K. (2017). Pleiotropic effects of statins on the cardiovascular system. Circ. Res. 120, 229-243. doi: 10.1161/CIRCRESAHA. 116.308537

Pavasini, R., Vieceli dalla Sega, F., Gallo, F., Passarini, G., Papi, A., Contoli, M., et al. (2017). Endothelial dysfunction and increased platelet reactivity in patients with acute coronary syndrome and undiagnosed COPD: insights into the SCAP trial. Eur. Respir. J. 50, 50-53. doi: 10.1183/13993003.01183-2017

Piepoli, M. F., Hoes, A. W., Agewall, S., Albus, C., Brotons, C., Catapano, A. L., et al. (2016). European Guidelines on cardiovascular disease prevention in clinical practice. Eur. Heart J. 37, 2315-2381. doi: 10.1093/eurheartj/ehw106

Pirro, M., Mannarino, M. R., Bianconi, V., Simental-Mendía, L. E., Bagaglia, F., Mannarino, E., et al. (2016). The effects of a nutraceutical combination on plasma lipids and glucose: a systematic review and meta -analysis of randomized controlled trials. Pharmacol. Res. 110, 76-88. doi: 10.1016/j.phrs.2016.04.021

Poli, A., Barbagallo, C. M., Cicero, A., Corsini, A., Manzato, E., Trimarco, B., et al. (2018). Nutraceuticals and functional foods for the control of plasma cholesterol levels. An Intersociety position paper. Pharmacol. Res. 134, 51-60. doi: 10.1016/j.phrs.2018.05.015

Ruscica, M., Gomaraschi, M., Mombelli, G., Macchi, C., Bosisio, R., Pazzucconi, F., et al. (2014). Nutraceutical approach to moderate cardiometabolic risk: results of a randomized, double-blind and crossover study with armolipid plus. J. Clin. Lipidol. 8, 61-68. doi: 10.1016/j.jacl.2013.11.003

Sata, M., and Walsh, K. (1998). Endothelial cell apoptosis induced by oxidized LDL is associated with the down-regulation of the cellular caspase inhibitor FLIP. J. Biol. Chem. 273, 33103-33106. doi: 10.1074/jbc.273.50.33103

Sever, P. S., Dahlöf, B., Poulter, N. R., Wedel, H., Beevers, G., Caulfield, M., et al. (2003). Prevention of coronary and stroke events with atorvastatin in hypertensive patients who have average or lower-than-average cholesterol concentrations, in the anglo-scandinavian cardiac outcomes trial-lipid lowering arm (ASCOT-LLA): a multicentre randomised controlled trial. Lancet 361, 1149-1158. doi: 10.1016/S0140-6736(03)12948-0

Silverman, M. G., Ference, B. A., Im, K., Wiviott, S. D., Giugliano, R. P., Grundy, S. M., et al. (2016). Association between lowering LDL-C and cardiovascular risk reduction among different therapeutic interventions. JAMA 316:1289. doi: 10.1001/jama.2016.13985

Stone, N. J., Robinson, J. G., Lichtenstein, A. H., Bairey Merz, C. N., Blum, C. B., Eckel, R. H., et al. (2014). 2013 ACC/AHA guideline on the treatment of blood cholesterol to reduce atherosclerotic cardiovascular risk in adults: a report of the american college of cardiology/american heart association task force on practice guidelines. Circulation 129, S1-S45. doi: 10.1161/01.cir.0000437738.63853.7a

Tejada, S., Pinya, S., del Mar Bibiloni, M., Tur, J. A., Pons, A., and Sureda, A. (2017). Cardioprotective effects of the polyphenol hydroxytyrosol from olive oil. Curr. Drug Targets 18, 1477-1486. doi: 10.2174/1389450117666161005150650
Trimarco, V., Izzo, R., Stabile, E., Rozza, F., Santoro, M., Manzi, M. V., et al. (2015). Effects of a new combination of nutraceuticals with morus alba on lipid profile, insulin sensitivity and endotelial function in dyslipidemic subjects. a cross-over, randomized, double-blind trial. High Blood Press Cardiovasc. Prev. 22, 149-154 doi: 10.1007/s40292-015-0087-2

Trpkovic, A., Resanovic, I., Stanimirovic, J., Radak, D., Mousa, S. A., CenicMilosevic, D., et al. (2015). Oxidized low-density lipoprotein as a biomarker of cardiovascular diseases. Crit. Rev. Clin. Lab. Sci. 52, 70-85. doi: 10.3109/ 10408363.2014.992063

Tsai, K.-L., Chen, L.-H., Chiou, S.-H., Chiou, G.-Y., Chen, Y.-C., Chou, H.-Y., et al. (2011). Coenzyme Q10 suppresses oxLDL-induced endothelial oxidative injuries by the modulation of LOX-1-mediated ROS generation via the AMPK/PKC/NADPH oxidase signaling pathway. Mol. Nutr. Food Res. 55, S227-S240. doi: 10.1002/mnfr.201100147

Valente, A. J., Irimpen, A. M., Siebenlist, U., and Chandrasekar, B. (2014). OxLDL induces endothelial dysfunction and death via TRAF3IP2: inhibition by HDL3 and AMPK activators. Free Radic. Biol. Med. 70, 117-128. doi: 10.1016/j. freeradbiomed.2014.02.014

Valgimigli, M., Agnoletti, L., Curello, S., Comini, L., Francolini, G., Mastrorilli, F., et al. (2003). Serum from patients with acute coronary syndromes displays a proapoptotic effect on human endothelial cells: a possible link to pan-coronary syndromes. Circulation 107, 264-270. doi: 10.1161/01.CIR.0000045665.57 256.86

Verhoeven, V., Van der Auwera, A., Van Gaal, L., Remmen, R., Apers, S., Stalpaert, M., et al. (2015). Can red yeast rice and olive extract improve lipid profile and cardiovascular risk in metabolic syndrome: a double blind, placebo controlled randomized trial. BMC Complement. Altern. Med. 15:52. doi: 10. 1186/s12906-015-0576-9

Vieceli Dalla Sega, F., Fortini, F., Aquila, G., Pavasini, R., Biscaglia, S., Bernucci, D., et al. (2018). Ticagrelor improves endothelial function by decreasing circulating epidermal growth factor (EGF). Front. Physiol. 9:337. doi: 10.3389/fphys.2018. 00337

Wang, S., Nie, S., and Zhu, F. (2016). Chemical constituents and health effects of sweet potato. Food Res. Int. 89, 90-116. doi: 10.1016/j.foodres.2016.08.032

Younes, M., Aggett, P., Aguilar, F., Crebelli, R., Dusemund, B., Filipiè, M., et al. (2018). Scientific opinion on the safety of monacolins in red yeast rice. EFSA J. 16:5368. doi: 10.2903/j.efsa.2018.5368

Zhang, Y., Kishi, H., and Kobayashi, S. (2018). Add-on therapy with traditional Chinese medicine: an efficacious approach for lipid metabolism disorders. Pharmacol. Res. 134, 200-211. doi: 10.1016/j.phrs.2018. 06.004

Conflict of Interest Statement: The authors declare that the research was conducted in the absence of any commercial or financial relationships that could be construed as a potential conflict of interest.

Copyright (C) 2019 Cimaglia, Vieceli Dalla Sega, Vitali, Lodolini, Bernucci, Passarini, Fortini, Marracino, Aquila, Rizzo, Ferrari and Campo. This is an open-access article distributed under the terms of the Creative Commons Attribution License (CC BY). The use, distribution or reproduction in other forums is permitted, provided the original author(s) and the copyright owner(s) are credited and that the original publication in this journal is cited, in accordance with accepted academic practice. No use, distribution or reproduction is permitted which does not comply with these terms. 